\title{
American Association for Cancer Research Genetics and Biology of Brain Cancers 2009, December 13-15, 2009, San Diego, CA
}

\author{
Isaac Yang $\cdot$ Linda M. Liau
}

Published online: 17 August 2010

(C) The Author(s) 2010. This article is published with open access at Springerlink.com

\begin{abstract}
Molecularly targeted therapies promise to transform the treatment of cancer patients, including those with brain tumors. A deeper understanding of the biology of brain tumors has led to a palpable excitement that new and more effective treatments are on the horizon for these deadly diseases. This conference brought basic, genomic, and translational scientists together with clinicians to discuss how to develop more effective molecularly targeted therapies for brain tumor patients based on a mechanistic understanding of the molecular circuitry and biology of the disease.
\end{abstract}

Keywords Brain tumor - Cancer - Glioblastoma . Glioma · Genomics

\section{Meeting:}

http://www.aacr.org/home/scientists/meetings-workshops/ special-conferences/genetics-and-biology-of-brain-cancers. aspx

\section{Organizers:}

Lynda Chin, M.D.

Dana-Farber Cancer Institute, Boston, MA

Webster K. Cavenee, Ph.D.

Ludwig Institute, University of California, San Diego, CA

I. Yang

UCSF, San Francisco, USA

L. M. Liau ( $\square)$

UCLA, Los Angeles, USA

e-mail: LLiau@mednet.ucla.edu
Paul S. Mischel, M.D.

University of California, Los Angeles, CA

Mitchel S. Berger, M.D.

University of California, San Francisco, CA

Keynote session: Defining the clinical problems

Chaired by Webster K. Cavenee (Ludwig Institute, University of California San Diego, San Diego, USA).

Advances in surgical management of gliomas

In this session, Mitchel S. Berger, (University of California San Francisco, San Francisco, USA) presented the data on how surgery for brain cancer can be safe, minimizing morbidity, and providing the scientific specimens for molecular and genetic study and analysis. He reviewed his experience with modern imaging modalities, language mapping, gross total resection of gliomas to demonstrate that gliomas can be resected safely. Until recently, dominant side craniotomies required generous craniotomy and a wide margin around eloquent cortex, but these advances have allowed for smaller craniotomy and more precise tumor resection around eloquent cortex [1]. An important point in his presentation was the observation that higher volume tumor centers will become more important as these centers are associated with decreased morbidity for tumor resections. Furthermore, Dr. Berger and colleagues have shown that aggressive surgical resection of low grade gliomas may have a significant improvement on survival outcomes and alter the natural history of malignant transformation of these low grade gliomas. This finding suggests that aggressive surgical resection may contribute 
significantly to improving patient survival [2]. Lastly, he suggested that targeted therapeutics may be most effective after aggressive and safe surgical resection which leaves the least amount of residual disease for the therapy to target.

\section{Current status of targeted therapies in malignant gliomas}

W. K. Alfred Yung (University of Texas M.D. Anderson Cancer Center, Houston, USA) discussed the current status of various targeted therapeutics against glioblastomas and indicated that there still remained significant improvements that have yet to be achieved. His summary of targeted therapeutics identified several key components of targeted treatments against brain cancer: (1) identifying the targets, (2) recognizing that these targets are drivers of cancer phenotype, (3) developing drugs against these targets, (4) improved biomarkers to characterize the presence of these targets, and (5) delivery of these focused drugs against these targets. He strongly suggested that targeted therapeutics have been less successful because of the myriad of molecular heterogeneity and overlapping regulatory pathways. His review of the EGFR and VEGF data suggested that the signaling and targeting of these signal pathways is complicated and not a simple task. His data from the M.D. Anderson group demonstrated in an animal model that anti-VEGF therapy may ultimately drive the tumor to develop a more invasive resistant phenotype. In summary, he recognized several obstacles to improving targeted therapies: (1) multiple activated pathways, (2) compensatory pathways, (3) resistant phenotypes, and (4) delivery mechanisms to the brain cancer. Future individualized targeted therapy designs with molecular surveillance imaging may lead to overcoming some of these obstacles against targeted therapeutics.

\section{Targeting signal transduction pathways in brain cancer}

In this session, Paul S. Mischel (University of California Los Angeles, Los Angeles, USA) discussed the important role that pathologists will play in identifying the underlying molecular and genetic defects that comprise glioblastomas. He reviewed the importance of knowing the critical parts of signaling pathways, but also suggested that knowing these critical steps in signal transduction pathways does not make altering these pathways any easier. Dr. Mischel suggested that the future of improved cancer genetic analysis will lead to personalized and individualized medicine based on the suite of molecular alterations present in each patient's tumor [3]. Dr. Mischel stressed the importance of integrating functional and genomic studies, citing the EGFR/PI3K signaling network as an example. He showed how attempts to inhibit EGFR/PI3/MTOR signaling in glioblastoma patients have failed due to PTEN loss, co-activation of other receptor tyrosine kinases, and activation of feedback loops to maintain signal flux through the PI3K pathway. Thus, understanding the critical components is not sufficient; understanding how they function together is needed. Interestingly, it was also discussed that intra-tumor cellular and molecular heterogeneity are potentially responsible for promoting resistance to targeted therapies [4], and the current suite of technologies is not well-suited to dissect this heterogeneity. He provided an example of one type of technology that can potentially be used to capture defined cell populations directly from clinical samples for in-depth molecular analysis.

\section{Special: Point-Counterpoint}

Chaired by Mitchel S. Berger, (University of California San Francisco, USA).

\section{EGFR targeting in malignant glioma: translational} and clinical implications

Dr. Michael Prados, (University of California San Francisco, San Francisco, USA) presented his point that early phase clinical trials have shown ambiguous results targeting EGFR and that these ambiguous results make it hard to know which targets to aim for and which modalities to utilize in chasing after these various targets. EGFR was identified to be amplified or over expressed in many gliomas, and pre-clinical and early phase trials led to the Phase 2 evaluation of erlotnib versus temozolomide or carmustine in recurrent glioblastoma. These brain tumor group clinical trials of erlotnib demonstrated no significant improvement in survival [5]. Retrospective evaluation of targeting EGFR has shown meager results, and the use of EGFR inhibitors has become less utilized by neuro-oncologists. This less then encouraging experience has raised doubts in the applicability of pre-clinical data to predict clinical applications and asks whether we have identified the critical prognostic factors or are utilizing inappropriate pre-clinical models for developing translational and developmental therapeutics.

Targeted therapy clinical trials in glioblastoma: an opportunity to learn from failure?

For the counterpoint, Dr. Timothy Cloughesy (University California Los Angeles, Los Angeles, CA) discussed that over the past decade, numerous investigators, and the TCGA, have provided whole genome evaluation of gene expression, DNA copy number and selected sequencing of 
genes which have clarified some of the altered cellular pathways in glioblastoma likely responsible for tumorigenesis and progression. The past decade also provided the opportunity to evaluate various targeted therapies focused upon inhibiting these altered signals. These clinical evaluations include inhibition of various receptor tyrosine kinases, as well as inhibiting, presumably important, downstream nodes of these receptors. However, with few exceptions, these investigations have failed to achieve stated clinical objectives (response rate, progression free survival, and overall survival), which has made the end goal of personalized medicine, based upon the molecular make up of the tumor, elusive [6]. Improving our understanding of these failed clinical evaluations will provide guidance toward the effective development of targeted agents in glioblastoma. To be successful, we will need to change our clinical evaluation paradigm to include obtaining tissue during drug delivery. Additionally, we need to develop technologies for improved tissue evaluations in order to anticipate molecular adaptations, feedback loops, and tumor heterogeneity, potentially on the single cell level. Finally, we need to promote the cooperation of drug developers toward the development of potent selective inhibitors and combination therapies.

\section{Session 1: Pediatric brain tumors: Developmental origin and clinical implications}

Chaired by Cameron W. Brennan (Memorial SloanKettering Cancer Center, New York, NY).

\section{Developmental biology of pediatric brain cancer}

Dr. David H. Rowitch (University of California San Francisco, San Francisco, CA) presented data indicating that the bHLH transcription factor Olig2 is critical and required for the gliomagenesis of astrocytomas. His data suggested that Olig2 is expressed in all adult and pediatric gliomas and is an inducible pathway for gliomagenesis by opposing the action of p53. Olig2 may interfere with the interactions of p53 binding to p21 cis acting DNA regulatory sequences. This may provide an attractive target for anti-tumor therapies by aiming for this molecular target of Olig2 [7]. Dr. Rowitch also reported some data from a large genomic study of pediatric tumors, which indicated that BRAF V600 mutations may play a critical role in gliomagenesis. His work with mice models suggests that BRAF V600 mutation coupling with Ink4a/Arf deletions may be obligatory to gliomagenesis. These findings may provide novel intracellular and signaling targets for the development of anti-tumor therapeutics for pediatric brain tumors.
Cellular and molecular origin of embryonal brain tumors

In this session, Dr. Charles G. Eberhart (Johns Hopkins University School of Medicine, Baltimore, MD) reviewed medulloblastoma and CNS PNET. Medulloblastomas appear to originate from both the external germinal layer (EGL) of the cerebellum and stem cells in other regions such as the ventricular zone. Tumors arising from the EGL generally feature high levels of Hedgehog signaling. In contrast, medulloblastoma with active Wnt signaling express ventricular zone markers and are associated with better clinical outcomes. In one recent analysis, both the Hedgehog and Wnt medulloblastoma subgroups showed signs of Notch activity, and crosstalk between Notch and Hedgehog may play a role in the emergence of therapeutic resistance when such pathways are targeted in medulloblastoma or glioblastoma. Additional medulloblastoma subgroups with poor clinical outcomes tend to have anaplastic histology and show elevated expression of MYCC and genes associated with phototransduction and semaphorin signaling. CNS PNET are also becoming more precisely subcategorized by paired microscopic and genetic analyses. One relatively common subtype, known as embryonal tumor with abundant neuropil and true rosettes (ETANTR), appears to be defined molecularly by an amplicon at chromosome 19q13 resulting in overexpression of specific microRNAs [8]. In summary, medulloblastoma and CNS PNET are molecularly heterogeneous and contain multiple genetically and clinically distinct subgroups. Developmentally significant signaling pathways are frequently activated in embryonal tumors and represent potential therapeutic targets. However, accurate molecular subclassification of embryonal tumor subtypes will be critical for the development of effective targeted therapies.

\section{Pediatric brain tumors: it is all about where they are born}

Dr. Richard J. Gilbertson (St. Jude Children's Research Hospital, Memphis, TN) reviewed the heterogeneity in clinically defined ependymomas and medulloblastomas that present a clinical challenge, especially in the pediatric population [9]. His data suggests that radial glia may play a critical role in the development of ependymomas and that recent transcriptomes of human ependymomas match specific variants of neural stem cells. Their animal model of a mouse ependymoma can model a human subgroup of ependymoma from which novel targets can be studied. Lastly, he reviewed the Wnt class of medulloblastomas which may arise from the ventricular region cells in contrast to the hedgehog activated, external germinal derived medulloblastoma. This Wnt induced pathway may represent a distinct subgroup of tumors which can be effectively modeled and molecularly targeted. His 
presentation highlighted the critical role that the CTNNB1 gene, through beta-catenin expression, likely induces hyperplasia and medulloblastoma formation through the Wnt signaling system.

\section{Defining the permissive microenvironment in pediatric glioma}

In his proffered talk, Dr. David H. Gutmann (Washington University School of Medicine, Saint Louis, MO) suggested that pediatric gliomas are markedly different from adult gliomas, because pediatric brain tumors are more related and similar to the steps of normal brain development in their response to extracellular signals. The cellular and molecular heterogeneity inherent in pediatric brain tumors highlight the importance of non-neoplastic elements in these tumors. His work on neurofibromatosis-1 (NF1) has led to a better understanding pediatric gliomas [10]. He reported that Nf1 loss of heterozygosity in the non-neoplastic tumor microenvironment provides a permissive condition in cooperation with complete Nf1 inactivation in glia to facilitate tumorigenesis. Moreover, he showed that resident microglia in these tumors are critical for tumor growth in genetically-engineered mice in vivo, supporting the notion that non-neoplastic stromal cells provide instructive cues important for brain tumor development and maintenance in children.

\section{Session 2: Adult brain tumors: Developmental origin and clinical implications}

Chaired by Paul S. Mischel (David Geffen School of Medicine at UCLA, Los Angeles, CA).

\section{Receptor signaling and tumor heterogeneity}

In this session, Dr. Webster K. Cavenee (Ludwig Institute, University of California San Diego, La Jolla, CA) postulated that tumor heterogeneity in glioblastoma is an active process driven by a mutant EGFR-induced paracrine circuit. His data from primary GBM samples showed that tumors expressing EGFRvIII usually do so in a small proportion of cells and are usually surrounded by tumor cells expressing wild-type EGFR [11]. In xenograft studies, this mutant EGFR enhanced the growth of wild-type EGFR-expressing tumor cells and suggested an active process of interaction between these two different subpopulations of tumor cells. By transferring media conditioned by mutant EGFR to wild-type EGFR cells, the same effect could be seen in wild-type EGFR growth and proliferation. Dr. Cavenee demonstrated that EGFR mutant cells do not secrete EGFR ligands, but rather secrete a STAT upregulating signal that can be inhibited if the cytokine receptor, GP130, is blocked. Hence this active interaction driven by the mutant EGFR receptor is a trans method for signaling though cytokines such as IL6 and LIF that also actively maintains this heterogeneity in glioblastoma. The smaller population of EGFR mutant cells can help drive the heterogeneity and growth of gliomas, and may represent a cancer cell-cell interaction that could be a target of therapeutic significance.

\section{Some human cancers have extraordinary high} frequencies of tumorigenic cells

Dr. Sean J. Morrison (University of Michigan Medical School, Ann Arbor, MI) presented data that suggest while some cancers follow the cancer stem cell model, other tumors may have common tumorigenic cells with little sign of hierarchical organization. He reported that some PTEN leukemias follow the cancer stem cell model with a small proportion of these cells being tumorigenic [12]. On the other hand, metastatic melanoma has a much higher frequency of tumorigenic cells and does not follow the cancer stem cell model. Heterogeneity is not necessarily a hallmark of hierarchy or the stem cell model, and cancers need not be hierarchically organized in order to be heterogeneous. Furthermore, he stated that tumor growth rate does not correlate with the frequency of tumorigenic cells in melanoma. With regard to gliomas, he hypothesized that there are intrinsic differences in cancers that are not relatable to stem cells. His preliminary data in mice suggested that tumorigenic cells are common in some glioblastomas similar to melanoma, and that CD133 did not distinguish between tumorigenic and non-tumorigenic cells in some of these tumors. Some glioblastomas may be similar to melanoma with a high percentage of nonstem-like tumorigenic cells.

\section{Dual role of primary cilia in medulloblastoma}

In this session, Dr. Arturo Alvarez-Buylla (University of California San Francisco, San Francisco, CA) reviewed the 2 neural stem cell regions in the subventricular zone and the hippocampus. His data revealing the pinwheel architecture of the ventricular surface was reported. Focusing on medulloblastoma development, his work focuses on the role of primary cilia in tumorigenesis and demonstrated the importance of cilia in the organization of stem cells and in mediation of sonic hedgehog signaling. Without cilia, radial astrocytes do not form and postnatal neurogenesis fails. Dr. Buylla postulates that cilia mediate a SmoM2dependant tumorigenesis, which is an upstream mediator of 
hedgehog signaling. Conversely, his data suggests that cilia suppress GLI2 dependant tumorigenesis, which is a downstream transcriptional activator of sonic hedgehog signaling. He reported that cilia can both mediate and suppress medulloblastoma formation, depending on the underlying oncogenic event and may represent an organelle that is a potential target for therapy.

Trim3, the human homolog of Drosophila brat, regulates neural differentiation, Myc expression, and growth properties of gliomas

Dr. Daniel Brat (Emory University School of Medicine, Atlanta, GA) reported on Human Tripartite motif protein (Trim3), the human homolog of brat in Drosophila [13]. In Drosophila, brat regulates asymmetric cell division of neuroblasts. When it is mutated, undifferentiated neuroblasts accumulate as a tumor-like mass. In Drosophila, brat appears to suppress $M y c$ as its mode of action, which may have correlates in human gliomas, since $M y c$ is upregulated in these tumors, at least partially following TP53 and PTEN mutations. Dr. Brat's group have also explored the brat homolog (Trim $3 b$ ) in a vertebrate zebra fish model in which Trim3 loss is also associated with a predominance of neuroblastic cells. Using Rembrandt data, it was shown that Trim 3 is often lost in human gliomas and its loss is associated with poor prognosis in low-grade gliomas. Trim3 appears to be a candidate tumor suppressor in gliomas, as Trim3 is often down regulated or absent in glioblastoma and its loss is associated with greater growth potential. Moreover, in vitro studies demonstrate that Trim3 overexpression represses $c$-Myc in glioma cell lines and inhibits proliferation [14].

\section{Session 3: Modeling brain tumors in vivo}

Chaired by W. K. Alfred Yung (UT M. D. Anderson Cancer Center, Houston, TX).

\section{Radiation resistance in brain tumors and the PI3K pathway}

In this session, Dr. Eric C. Holland (Memorial SloanKettering Cancer Center, New York, NY) focused specifically on a side population of glioblastoma that is composed of endothelial and stem cells [15]. This glioblastoma subpopulation is enriched with neurosphereforming cells and is increased with PTEN loss. It also appears that through selection, temozolomide increases this phenotype of treated glioblastoma cells. It appears that this PDGF+ side-population of glioblastoma cells are highly concentrated near blood vessels, and it was suggested that this stem-cell likeness could be driven by the microenvironment of the peri-vascular niche. Some preliminary data suggests that eNOS and active Notch1 are both upregulated in PDGF gliomas and that perhaps nitric oxide may activate the notch signaling pathways in this subset of gliomas. Dr. Holland suggested that nitric oxide drives the side population phenotype in PDGF gliomas. Further work has shown that nitric oxide functions through cGMP to activate PKG and enhance the side population and notch signaling and can also be blocked by PKG inhibitors. Recent work has identified several GBM subtypes that can be defined by signaling characteristics, and the Proneural subgroup is most consistent with gliomas driven by PDGF. This subset of glioma tumor cells may identify new molecular targets relating to chemotherapy/radiation resistance in cells near the tumor vasculature. This nitric oxide notch pathway needs to be further evaluated in EGFR + tumors, as well as other glioblastoma subtypes.

\section{Glioma stem cell and animal models}

Dr. Luis F. Parada (UT Southwestern Medical Center, Dallas, TX) discussed how animal models can provide insights into the behavior of gliomas and glioma stem cells, particularly genetic animal models. Ablation of $N F 1 / p 53$ and PTEN can drive the formation of high-grade gliomas in a mouse model; but interestingly, early lesions are associated with the ventricles and the peri-ventricular space in pre-symptomatic mice. Whereas the targeting of cells away from the ventricular zone fails to induce gliomas, genetic alterations such as NF1, p53, and PTEN deletions in the stem-like cells in the ventricular zone can successfully induce gliomas. Dr. Parada suggested that xenograft animal models may greatly underestimate the percentage of tumor initiating cells, and that these assays may not reflect the physiology of tumors in the normal microenvironment. Accurate and physiologically relevant genetic animal models present an alternative to study and gain insights on gliomas and glioma stem cells. Further work suggests that both neural stem cells and glial progenitor cells can be genetically altered to form gliomas [16]. There are many lessons to learn from physiologically relevant animal models of cancer, and it appears that the stem cell progenitor compartment is a likely source of many brain tumors.

\section{A glioblastoma model using lentiviral vectors}

In this proffered presentation, Dr. Dinorah FriedmannMorvinski (Salk Institute for Biological Studies, La Jolla, CA) presented work on a novel glioma mouse model. This lentiviral inducible mouse model in adult immunocompetent mice is created by injecting Cre-loxP-controlled lentiviral vectors expressing oncogenes. GFAP region-specific 
cells were utilized to develop this glioblastoma model. The subventricular zone and hippocampus were readily induced to form glioblastoma when transduced, but the cortex previously was not a commonly a site for inducible glioblastoma. Transplantation of brain tumor cells which contained CD133 + cells formed tumorspheres and could differentiate into neurons and astrocytes when transplanted into naïve mice brains and subsequently formed glioblastoma-like tumors [17]. Other studies are needed to evaluate if CD133 is the best marker for these transplantable cells in this animal model. Some data suggests that CD15 may be a more appropriate identifier for this subset of glioma cells. This presentation suggested that Cre-loxP-controlled lentiviral vectors can generate a mouse glioblastoma model in a region- and cell type-specific manner. Dr. FriedmannMorvinski also shared results with their new lentiviral vector that expresses both the oncogene H-Ras and the tumor suppressor sip53. Improving on their previous work, they can now obtain glioblastoma-like tumors in all three locations that were injected: hippocampus, sub-ventricular zone and cortex.

\section{Manipulating Drosophila glia to model human glioma}

Dr. Renee D. Read (Salk Institute for Biological Studies, La Jolla, CA) reviewed that Drosophila brain gene expression is homologous to mammalian brain and can lead to new insights. Activations of EGFR, RAS, and PI3K cause neoplasia, but co-activation of EGFR and PI3K does not elicit overgrowth from all neural cell types. Constitutive co-activation of EGFR-Ras and PI3K pathways in Drosophila glia and glial precursors gives rise to neoplastic, invasive glial cells that create transplantable tumors that model human glioma [18]. Further data demonstrates that EGFR and $P I 3 K$ mutations synergistically activate several signaling pathways that lead to glioma induction in Drosophila glial cells. These mechanisms contribute to cell cycle entry, protein expression, and migration for these Drosophila glial cells, which may represent a model for identifying novel homologous human targets [18]. Lastly, this live whole-body Drosophila model may permit pilot screening of compounds and evaluation of novel drugs for therapeutic efficacy.

\section{Session 4: Genomics and genetics of brain cancers}

Chaired by Antonio Iavarone (Columbia University, New York, NY).

\section{Functionalizing the cancer genome}

In this session, Dr. Lynda Chin (Dana-Farber Cancer Institute, Boston, MA) reviewed the complex nature and the redundancy in the function of various genetic alterations and signaling pathways in glioma [19]. She discussed the various patterns of $R b$ pathway inactivation in glioma and noted that redundancy is the norm. Furthermore, she showed that the specific pattern of $\mathrm{Rb}$ pathway inactivation can be correlated with sensitivity to selective inhibitors targeting core components of the $\mathrm{Rb}$ pathway, such as CDK4/6. Other genetic mutations such as NF1 and p53 have been identified to be significant in glioblastoma, and the cancer genome analysis provides a systematic and comprehensive analysis of the genetic alterations and signaling pathways critical in the biology of glioblastoma. Although there are a large number of candidates emerging from large-scale cancer genome efforts such as TCGA, functional validation and mechanical insight are required to cull bystanders from drivers and to better understand the biological relevance of these genomic alterations. Highthroughput functional validation and context-specific analysis is required to functionalize the cancer genome because of the extreme heterogeneity and complexity of the glioblastoma cancer genome.

\section{Developing targeted therapies for glioblastoma}

Dr. Heidi S. Phillips (Genentech, Inc., South San Francisco, $\mathrm{CA})$ reviewed the prognostic subclasses of glioma: the proneural, mesenchymal, and proliferative subtypes [20]. These subtypes have prognostic significance, as overall survival is poor in the proliferative and mesenchymal groups and best in the proneural group. As novel targeted therapies such as anti-angiogenic therapies are developed, these biomarkers may identify which patients may be most ideal for these therapies. To further advance the development of glioma-targeted therapies, improved glioma models must be developed. Tumor xenografts with their clear borders do not accurately mimic infiltrative gliomas. Although neurosphere grafts are invasive glioma models, these tumor models lack angiogenesis, EGFR amplification and mesenchymal markers such as $Y K L 4 O$. There is a need to develop novel glioma models that demonstrated both a diffuse invasion pattern and the angiogenic phenotype. Lastly, Dr. Phillips emphasized that the proneural group with younger age and improved outcomes appear to be correlated with the prognostic factor of IDHI mutation.

Functional analysis of glioblastoma kinase mutations found in TCGA: implications for targeted therapy

Dr. Matthew L. Meyerson (Dana-Farber Cancer Institute, Boston MA) reviewed the importance of glioma therapeutic targets identified from the TCGA analysis. It may be critically important in order to evaluate the benefit of target alteration to genomically screen, select, and stratify 
patients in the analysis of clinical trials for novel therapeutics. For example, in the analysis of EGFR kinase, which is amplified in many gliomas [21], the EGFR inhibitors were not found to be effective in glioma clinical trials, which were not stratified according to the presence or absence of variations in EGFR expression. Nevertheless, these kinases function as significant regulators of cellular activity and represent critical targets for anti-tumor therapy. Genomic stratification will identify which targeted therapy will be most effective for which patients, and should be used as an approach to stratify patients for clinical trials. Also, the analyses of the TCGA results may identify new targets for therapy against glioma, such as the $\mathrm{C}$ terminus mutations in EGFR, NF1, PI3K, and AKT. Genomic analysis may help identify which patients can benefit from EGFR kinase inhibitors or other targeted therapies in the clinical trials of glioblastoma.

\section{An analysis of copy-number changes across multiple cancer types}

In this talk, Dr. Rameen Beroukhim (Dana-Farber Cancer Institute, Boston, MA) evaluated for common genetic events across multiple cancer types. Over 2520 tissue samples and 611 cell lines were all systematically analyzed using a 250,000 single nucleotide polymorphism (SNP) array platform in a high throughput fashion. Because SNPs are common genetic variations in the genome, they can be useful as genomic markers. This analysis found 82 deleted regions and 76 amplified regions, which were common among various cancer types. Many deletions tended to affect the largest genes in the genome. Amplifications and deletions often involved $B C L 2$ family members, including $M C L 1$ and $B C L 2 L 1$. Focal genetic events tend to be shared across multiple cancer types. These copy number data will be shared with the neuro oncology community on the website www.broadinstitute.org in order to improve the efficiency and limit the research effort redundancy. We commend these efforts to increase the speed of information dissemination, and the collaboration that tumorscape will foster may lead to improved efficiencies in brain tumor research [22].

\section{Inferring miRNA-mRNA regulatory networks in glioblastoma}

In the final proffered presentation of the afternoon, Dr. Sachet A. Shukla (Dana-Farber Cancer Institute, Boston, MA) utilized the context of likelihood of relatedness (CLR) algorithm, an extension of the relevance networks class of algorithm [23], to evaluate the relationship between miRNA and mRNA expression using TCGA glioblastoma genomic data. Using 194 TCGA glioblastoma samples with matched miRNA and mRNA expression, CLR analysis defined many novel associations, some specific to molecular subtypes defined by transcriptome profiling. When filtered with copy number data, several miRNAs emerged with strong evidence of links to key regulatory pathways in glioma. For example, consistent with recent publication (PMID: 19773441), microRNA 34a was related to the Notch signaling pathway in the regulatory network of glioblastoma. In summary, CLR network analysis can identify and infer transcriptional and regulatory interactions in glioblastoma.

\section{Session 5: Translational neuro-oncology: Targeted therapies and resistance}

Chaired by Terrance G. Johns (Monash Institute of Medical Research, Clayton, VIC, Australia).

Targeting immune cells, cancer stem cells, and metabolites in brain tumors

In this session, Dr. Linda M. Liau (University of California Los Angeles, Los Angeles, CA) began by defining the clinical problem of glioblastoma and the difficulty in treating the remaining infiltrating cells after surgical resection. She reviewed the results from a clinical trial of a dendritic cell-based vaccine for glioma [24] and found that overall survival was correlated with induction of a cytotoxic T-cell (CTL) responses and decreased TGF- $\beta 2$ expression [25]. In addition, dendritic cells can be optimized utilizing a toll-like receptor (TLR-7) agonist, imiquimod (Aldara ${ }^{\mathrm{TM}}$ ), which promotes dendritic cell survival, trafficking, and tumor-specific activation of T cells [26]. In 22 glioblastoma patients treated with a DC-based vaccine in conjunction with TLR agonists, the median survival was 1049 days, which is significantly greater than historical controls in this unblinded, non-randomized clinical trial. Interestingly, this trial did not have a selection bias towards better outcome genotypes as only 6 out of the 22 patients had a proneural gene expression pattern, with the remainder having the poorer prognosis proliferative and mesenchymal phenotypes. It appears that the mesenchymal subtypes had the most significant improvement in overall survival after dendritic cell vaccine therapy. Dr. Liau also presented data regarding the resistance of glioma stem cells to conventional therapies and the recent finding that $I D H I$ mutant gliomas are associated with a putative oncometabolite, 2-hydroxyglutarate, which may represent a potential new target for therapy that can be monitored using advanced MR spectroscopic analysis. 


\section{Targeting VEGF and integrins in malignant glioma}

In this presentation, Dr. Roger Stupp (University of Lausanne, Switzerland) focused on the clinician's view of targeted therapy and how targeting angiogenesis has translated into clinical applications. Anti-VEGF therapy appears to normalize vascular permeability [27], translating into rapid decrease of edema and radiographic responses (decrease in contrast enhancement). However, the tumor will activate alternative pathways and escape mechanisms (invasion and migration) and may subsequently demonstrate a more aggressive phenotype [28]. These mechanisms require further elucidation, and combined blockage of multiple pathways may be required. Discontinuation of anti-VEGF therapy may be associated with a rebound phenomenon leading to rapid clinical deterioration. Dr. Stupp also discussed integrins, which are heterodimeric transmembrane proteins that mediate migration and attachment, as potential targets for antiglioma therapy. Cilengitide, a selective integrin inhibitor, has been shown to decrease invasiveness in vitro and shows synergy with radiation and with chemotherapy in an animal model. Initial clinical data in recurrent and newly diagnosed glioblastoma is promising, and larger randomized trials are ongoing. Many of these targeted and anti-angiogenic agents may work best in combination with chemotherapy. As MGMT promoter methylation is an indicator for sensitivity to alkylating agent chemotherapy, determination and stratification or selection according to the MGMT methylation status is of importance $[29,30]$.

\section{PTEN tyrosine phosphorylation as a determinant of response to EGFR inhibitors in glioblastoma}

In this presentation, Dr. Tim Fenton (Ludwig Institute, University of California San Diego, La Jolla, CA) reviewed the relationship between EGFR and PTEN in gliomas [31], and that loss of PTEN expression in GBM attenuates responsiveness to EGFR kinase inhibitors. Cases of gliomas resistant to EGFR inhibitors despite having wild-type PTEN could be explained by post-translational impairment of PTEN. This group reported that PTEN phosphorylation is correlated to Sac activation, which may demonstrate a potential mechanism for post-translational modification of PTEN and for resistance to EGFR inhibitors despite a wildtype PTEN genotype. Inhibition of the Src pathway with a drug such as Dasatnib may increase the sensitivity and response to EGFR inhibitors in gliomas. Restoration of PTEN or modifying this post-translational alteration of PTEN may mitigate EGFR inhibitor resistance in wild-type PTEN glioma.
Mechanisms of evasive resistance to VEGF inhibition in mouse models of glioblastoma

In her presentation, Dr. Gabriele Bergers (University of California San Francisco, San Francisco, CA) presented data that hypoxia through HIF1-alpha is commonly present in glioblastomas and stimulates the recruitment of vascular progenitor cells and pro-angiogenic myeloid cells to the tumor site, which facilitates neovascularization [32]. Although blocking neovascularization has demonstrated encouraging results in glioblastoma patients, a clinical challenge is the rebound and increased invasiveness after anti-VEGF therapy. Blocking the VEGF pathway inhibits angiogenesis, increases tumor apoptosis, and may increase survival, but it also increases the invasiveness of glioma [32]. VEGF may limit invasiveness of the tumor through VEGF receptor-mediated effects. In mouse models of glioblastoma, anti-VEGF therapy may elicit tumor adaptation and tumor progression to stages of greater malignancy, with increased invasiveness and occasional distant metastasis [32]. Ablating the VEGF receptor also increases tumor invasiveness. Thus, anti-VEGF therapies may alter the natural history of gliomas into a more invasive and aggressive phenotype after anti-angiogenic therapy. This alteration warrants further studies, as the use of anti-angiogenic therapies continue to increase in glioma treatment.

\section{Pathway analysis of glioblastoma tissue after pre-operative treatment with the EGFR tyrosine kinase inhibitor gefitinib}

In this proffered talk, Dr. Monika E. Hegi (University Hospital Lausanne, Lausanne, Switzerland) evaluated the effect of gefitinib on glioma. Epidermal growth factor receptor (EGFR) is frequently amplified and overexpressed in high-grade gliomas and represents an intriguing therapeutic target [33]. However, clinical trials with EGFR inhibitors have not found significant impact in the treatment of high-grade gliomas, raising the question if the drugs actually reach the target. Gefitinib acts as an ATP mimetic agent, binding to the cytoplasmic ATP pocket domain and blocking receptor phosphorylations and EGFR mediated activation of downstream pathways. In patients treated preoperatively with gefitinib, high drug levels were reached in the glioblastoma tissues and EGFR phosphorylation was efficiently blocked. However, phosphorylation of key downstream pathway constituents such as $A K T$ seemed not affected, suggesting that the activity of the pathway may not be efficiently inhibited. Redundancy of the pathway and negative feed-back loops may contribute to this effect. Further studies are required to identify key players involved in this treatment resistance in order to design effective combination therapies for successful attenuation of the pathway. 
Targeting RTK-induced oxidative stress by PARPI inhibition in glioblastoma therapy

In the final presentation of this session, Dr. Clark C. Chen (Dana-Farber Cancer Institute, Boston, MA) suggested that EGRv3 over-expression may manifest in increased reliance on DNA repair pathways. Radiation introduces DNA damage and may be optimized using siRNA against DNA repair genes. Poly-(ADP-ribose) polymerase (PARP1) catalyzes the transfer of ADP and mediates DNA repair. Inhibition of PARP1 through siRNA demonstrates increased radiosensitization of EGFRv3-overexpressing U87 glioma cells in vitro. This mechanism may be due to the increased accumulation of reactive oxygen species in EGFR v3 overexpressing cells. EGFR hyperactivity may induce DNA damage accumulation, which is enhanced by PARP1 inhibition. Interestingly, high expression of PARP1 trends with poorer survival among EGFR-amplified and or EGFRmutated tumors. RNA interference (RNAi) of DNA repair pathways may optimize radiation and chemotherapy adjuvants again gliomas with further investigation. Highthroughput genetic screening may identify DNA repair pathways that might contribute to uncovering glioma vulnerabilities [34].

\section{Session 6: Translational neuro-oncology: drug delivery and imaging}

Chaired by Darell D. Bigner (Duke Comprehensive Cancer Center, Durham, NC).

\section{Advances in imaging of patients with brain tumors}

In this presentation, Dr. Sarah J. Nelson (UCSF Comprehensive Cancer Center, San Francisco, CA) reviewed the potential for non-invasive biomarkers for assessing tumor properties. Her research is currently focusing on imageguided tissue collection with ex vivo analysis, which allows correlation of imaging markers with tissue biologic analysis. Structural, vascular, and metabolic imaging modalities are required to develop non-invasive biomarkers for assessing treatment effects after radiation, chemotherapy, and other novel glioma therapies. Diffusion imaging can be utilized to identify tumor infiltration and distinguish tumor recurrence from treatment effect. Dr. Nelson's data suggested that higher apparent diffusion coefficient (ADC) on diffusion MRI correlates with both increased tumor infiltration and cellularity, which results in worse prognosis [35]. Vascular parameters and metabolites, such as choline and lactate, are other useful biomarkers for prognosis and glioma progression. Future application of MR metabolic imaging using hyperpolarized C-13-enriched agents may be a promising new technology for radiographic evaluation of glioblastoma [36]. Utilizing dynamic nuclear polarization in this manner has enabled the direct monitoring of $\mathrm{C}-13$ metabolites in vivo at a high signal-to-noise ratio and may improve both the sensitivity and specificity of imaging for assessing treatment effects in patients with glioma.

\section{Potential of RNAi therapeutics for the treatment of glioblastoma}

In this session, Dr. Dinah Sah (Alnylam Pharmaceuticals, Cambridge, MA) reviewed the feasibility of utilizing RNA interference (RNAi) to inhibit any molecular target of interest. Synthetic small interfering RNAs (siRNAs) harness the endogenous RNAi pathway, and can be designed to silence any gene through the cleavage and degradation of its mRNA. After silencing a target with siRNA, the durability of this effect appears to last for approximately 3 weeks. In vivo studies have demonstrated the ability of lipid nanoparticle-formulated siRNAs to reduce tumor burden in an orthotopic liver tumor model in mice, suppress the targeted mRNAs, and produce the expected mRNA cleavage products, confirming the RNAi mechanism. CNS molecular targets have also been suppressed in rats and non-human primates with direct intraparenchymal brain delivery of siRNA, which suggests the potential of this approach for anti-tumor therapy in gliomas.

\section{Cancer-associated IDH1 mutations produce 2-hydroxyglutarate}

In the final presentation, Dr. Mark A. Bittinger (Agios Pharmaceuticals, Cambridge, MA) reviewed that the enzyme, isocitrate dehydrogenase 1 (IDH1) converts isocitrate to alpha-ketoglutarate, and that $I D H 1$ mutations are commonly found in low-grade gliomas and secondary glioblastomas while relatively rare in primary glioblastomas. IDH1 mutations are single base pair mutations that mutate arginine 132 to histidine, and the mutant enzyme has previously been demonstrated to harbor impaired oxidative decarboxylation activity. Cells expressing mutant IDHI were found to contain elevated levels of the metabolite 2-hydroxyglutarate [37]. This elevation of 2-hydroxyglutarate was shown to be due to a novel enzymatic activity of mutant $I D H 1$, which converts alpha-ketoglutarate into 2-hydroxyglutarate. Interestingly, increased 2-hydroxyglutarate levels have been shown to correlate with an increased risk of malignant brain tumors in patients with inborn errors of 2-hydroxyglutarate metabolism [37]. IDH1 mutations are correlated with high levels of 2-hydroxyglutarate in human gliomas and 2-hydroxyglutarate may be a biomarker with important clinical value for prognosis, as well as a tractable therapeutic target. 
Open Access This article is distributed under the terms of the Creative Commons Attribution Noncommercial License which permits any noncommercial use, distribution, and reproduction in any medium, provided the original author(s) and source are credited.

\section{References}

1. Sanai N, Mirzadeh Z, Berger MS (2008) Functional outcome after language mapping for glioma resection. $\mathrm{N}$ Engl $\mathrm{J}$ Med 358(1):18-27

2. Smith JS, Chang EF, Lamborn KR et al (2008) Role of extent of resection in the long-term outcome of low-grade hemispheric gliomas. J Clin Oncol 26(8):1338-1345

3. Thomas RK, Baker AC, Debiasi RM et al (2007) Highthroughput oncogene mutation profiling in human cancer. Nat Genet 39(3):347-351

4. Bailey RC, Kwong GA, Radu CG et al (2007) DNA-encoded antibody libraries: a unified platform for multiplexed cell sorting and detection of genes and proteins. J Am Chem Soc 129(7): 1959-1967

5. van den Bent MJ, Brandes AA, Rampling R et al (2009) Randomized phase II trial of erlotinib versus temozolomide or carmustine in recurrent glioblastoma: EORTC brain tumor group study 26034. J Clin Oncol 27(8):1268-1274

6. Cloughesy TF, Yoshimoto K, Nghiemphu P et al (2008) Antitumor activity of rapamycin in a Phase I trial for patients with recurrent PTEN-deficient glioblastoma. PLoS Med 5(1):e8

7. Ligon KL, Huillard E, Mehta S et al (2007) Olig2-regulated lineage-restricted pathway controls replication competence in neural stem cells and malignant glioma. Neuron 53(4):503-517

8. Li T, Wen H, Brayton C et al (2007) Epidermal growth factor receptor and notch pathways participate in the tumor suppressor function of gamma-secretase. J Biol Chem 282(44):32264-32273

9. Ridley L, Rahman R, Brundler MA et al (2008) Multifactorial analysis of predictors of outcome in pediatric intracranial ependymoma. Neuro Oncol 10(5):675-689

10. Gutmann DH (2008) Using neurofibromatosis-1 to better understand and treat pediatric low-grade glioma. J Child Neurol 23(10): 1186-1194

11. Lu KV, Zhu S, Cvrljevic A et al (2009) Fyn and SRC are effectors of oncogenic epidermal growth factor receptor signaling in glioblastoma patients. Cancer Res 69(17):6889-6898

12. Reya T, Morrison SJ, Clarke MF et al (2001) Stem cells, cancer, and cancer stem cells. Nature 414(6859):105-111

13. Boulay JL, Stiefel U, Taylor E et al (2009) Loss of heterozygosity of TRIM3 in malignant gliomas. BMC Cancer 9:71

14. Betschinger J, Mechtler K, Knoblich JA (2006) Asymmetric segregation of the tumor suppressor brat regulates self-renewal in Drosophila neural stem cells. Cell 124(6):1241-1253

15. Bleau AM, Hambardzumyan D, Ozawa T et al (2009) PTEN/ PI3K/Akt pathway regulates the side population phenotype and ABCG2 activity in glioma tumor stem-like cells. Cell Stem Cell 4(3):226-235

16. Alcantara Llaguno S, Chen J, Kwon CH et al (2009) Malignant astrocytomas originate from neural stem/progenitor cells in a somatic tumor suppressor mouse model. Cancer Cell 15(1):45-56

17. Marumoto T, Tashiro A, Friedmann-Morvinski D et al (2009) Development of a novel mouse glioma model using lentiviral vectors. Nat Med 15(1):110-116

18. Read RD, Cavenee WK, Furnari FB et al (2009) A drosophila model for EGFR-Ras and PI3K-dependent human glioma. PLoS Genet 5(2):e1000374

19. McLendon R, Friedman A, Bigner D et al (2008) Comprehensive genomic characterization defines human glioblastoma genes and core pathways. Nature 455(7216):1061-1068
20. Phillips HS, Kharbanda S, Chen R et al (2006) Molecular subclasses of high-grade glioma predict prognosis, delineate a pattern of disease progression, and resemble stages in neurogenesis. Cancer Cell 9(3):157-173

21. Lee JC, Vivanco I, Beroukhim R et al (2006) Epidermal growth factor receptor activation in glioblastoma through novel missense mutations in the extracellular domain. PLoS Med 3(12):e485

22. Li C, Beroukhim R, Weir BA et al (2008) Major copy proportion analysis of tumor samples using SNP arrays. BMC Bioinformatics 9:204

23. Faith JJ, Hayete B, Thaden JT et al (2007) Large-scale mapping and validation of Escherichia coli transcriptional regulation from a compendium of expression profiles. PLoS Biol 5(1):e8

24. Liau LM, Black KL, Martin NA et al (2000) Treatment of a patient by vaccination with autologous dendritic cells pulsed with allogeneic major histocompatibility complex class I-matched tumor peptides. Case report. Neurosurg Focus 9(6):e8

25. Liau LM, Prins RM, Kiertscher SM et al (2005) Dendritic cell vaccination in glioblastoma patients induces systemic and intracranial T-cell responses modulated by the local central nervous system tumor microenvironment. Clin Cancer Res 11(15): $5515-5525$

26. Prins RM, Craft N, Bruhn KW et al (2006) The TLR-7 agonist, imiquimod, enhances dendritic cell survival and promotes tumor antigen-specific $\mathrm{T}$ cell priming: relation to central nervous system antitumor immunity. J Immunol 176(1):157-164

27. Batchelor TT, Sorensen AG, di Tomaso E et al (2007) AZD2171, a pan-VEGF receptor tyrosine kinase inhibitor, normalizes tumor vasculature and alleviates edema in glioblastoma patients. Cancer Cell 11(1):83-95

28. Brandes AA, Stupp R, Hau P et al (2010) EORTC study 2604122041: phase I/II study on concomitant and adjuvant temozolomide (TMZ) and radiotherapy (RT) with PTK787/ZK222584 $(\mathrm{PTK} / \mathrm{ZK})$ in newly diagnosed glioblastoma. Eur $\mathrm{J}$ Cancer 46(2):348-354

29. Weller M, Stupp R, Reifenberger G et al (2010) MGMT promoter methylation in malignant gliomas: ready for personalized medicine? Nat Rev Neurol 6(1):39-51

30. van den Bent MJ, Dubbink HJ, Sanson M et al (2009) MGMT promoter methylation is prognostic but not predictive for outcome to adjuvant PCV chemotherapy in anaplastic oligodendroglial tumors: a report from EORTC Brain Tumor Group Study 26951. J Clin Oncol 27(35):5881-5886

31. Furnari FB, Fenton T, Bachoo RM et al (2007) Malignant astrocytic glioma: genetics, biology, and paths to treatment. Genes Dev 21(21):2683-2710

32. Du R, Lu KV, Petritsch C et al (2008) HIF1alpha induces the recruitment of bone marrow-derived vascular modulatory cells to regulate tumor angiogenesis and invasion. Cancer Cell 13(3): 206-220

33. Brandes AA, Franceschi E, Tosoni A et al (2008) Epidermal growth factor receptor inhibitors in neuro-oncology: hopes and disappointments. Clin Cancer Res 14(4):957-960

34. Kahle KT, Kozono D, Ng K et al (2010) Functional genomics to explore cancer cell vulnerabilities. Neurosurg Focus 28(1):E5

35. Khayal IS, McKnight TR, McGue C et al (2009) Apparent diffusion coefficient and fractional anisotropy of newly diagnosed grade II gliomas. NMR Biomed 22(4):449-455

36. Hu S, Lustig M, Balakrishnan A et al (2009) 3D compressed sensing for highly accelerated hyperpolarized (13)C MRSI with in vivo applications to transgenic mouse models of cancer. Magn Reson Med 16:16

37. Dang L, White DW, Gross S et al (2009) Cancer-associated IDH1 mutations produce 2-hydroxyglutarate. Nature 462(7274): 739-744 